\title{
DETERMINATION OF THE MAGNETIC FIELD DISTRIBUTION IN THE Bi2212 SUPERCONDUCTING SINGLE CRYSTAL BY THE SCANNING EPR-PROBE
}

\author{
H. Drulis, L. Folcik, M. Drulis, A. Zaleski \\ W. Trzebiatowski Institute of Low Temperature and Structure Research \\ Polish Academy of Sciences, Okólna 2, 50-950 Wrocław, Poland \\ AND N.M. SulEimanov \\ Kazan Physicotechnical Institute, Russian Academy of Sciences \\ Sibirsky Tract 10/7, 420029 Kazan, Russia
}

The profile of the magnetic field distribution over the surface of superconducting sample is studied by the scanning an electron paramagnetic resonance microprobe. The magnetic field distribution is mapped from the measurements of the spin probe EPR-signal shift.

PACS numbers: 74.72.-h, 76.30.-v

\section{Introduction}

The magnetic flux distribution in the type II superconductors determines critical state of these materials and depends highly on the sample shape and on its orientation relative to the applied field. To reveal the vortex pattern on the Abrikosov lattice scale the Bitter technique [1] has been applied. On larger length scales the flux distribution can be observed by the Faraday method using an active magneto-optical layer [2]. Both methods provide the direct imaging of the magnetic-field distribution at the surface of the superconductors. To study the flux distribution within the bulk of the superconductors the nuclear magnetic resonance (NMR) and muon-spin resonance ( $\mu \mathrm{SR}$ ) are often used [3, 4]. Frait et al. [5] have proposed an electron-paramagnetic-resonance (EPR) method in which the local magnetic field intensities are probed by magnetic resonance of paramagnetic organic radicals such as DPPH, TCNQ, $\mathrm{Phc}_{2}$, etc. For the first time they used this method for a direct observation of the expulsion of the magnetic induction out of the superconductor sample due to the Meissner effect. Since then, it has been used by other authors for measurements of flux distribution associated 
with the Abrikosov lattice [6], grain structure [7], or the shape of the high $T_{\mathfrak{c}}$ superconductor samples [8].

The main goal of this paper is the determination of the magnetic field distribution near the Bi2212 single crystal in a magnetic field parallel to the $c$ axis: In the paper we analyze the long-distance (macroscopic) limit, where all short-wa velength distribution sources are averaged out and the position of the ESR line probes the mean value of the magnetic induction close to the surface. The point-to-point mapping is performed by using the movable EPR microprobe. For the first time such measurements have been presented by Kazan group for $\mathrm{Y}-\mathrm{Ba}-\mathrm{Cu}-\mathrm{O}$ superconducting sample [9]. Scanning the microprobe over the superconducting sample in external magnetic field, we will determine the field induction at a given point from the ESR-signal shift.

\section{Experimental}

The Bi2212 single crystals used in our studies were grown using conventional flux technique and had a shape of flat strip with the dimensions of $3 \times$ $1.5 \times 0.45 \mathrm{~mm}^{3}$. The superconducting transition temperature determined from the AC susceptibility in the magnetic field of $3.3 \mathrm{kOe}$ were relatively low $\left(T_{\mathrm{c}}=27 \mathrm{~K}\right)$. As an EPR probe the bisphtalocyanin-indium $\left(\mathrm{Pc}_{2} \mathrm{In}\right)$ free radical was used with the dimensions of $0.3 \times 0.3 \times 0.05 \mathrm{~mm}^{3}$. The original (undistorted) ESR spectrum of the probe consists of one narrow line of the Lorentz shape with isotropic $g$-factor equal to 2.003 and constant width of $\delta H=2.3$ Oe at $T=17 \mathrm{~K}$. The schematic diagram of the experimental device and the design details are displayed in Fig. 1.

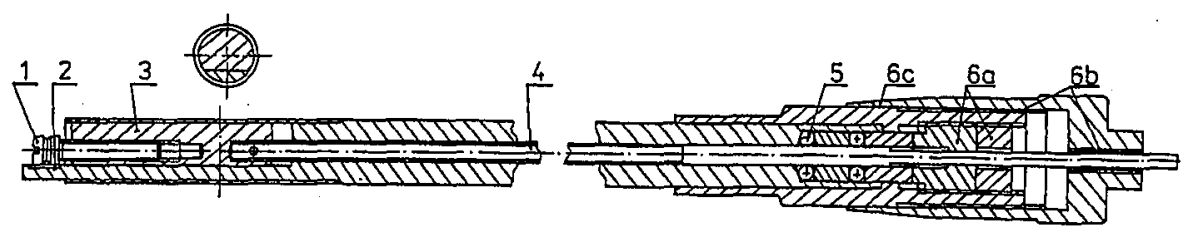

Fig. 1. Schematic sketch of the sample holder for the ESR tomography. 1 - adjusting screw with ESR probe - $Z$-axis; 2 - the sample spot; 3 - movable part - Y-axis; 4 - stainless steel rode; 5 - O-ring; 6 - micrometer screw $1 / 100 \mathrm{~mm}$.

The sample was fixed with glue on the solid part (2) of the holder whereas the probe was glued to the movable one (3). During the experiment the ESR probe was shifted over the flat crystal surface along the $Y$-axis as is shown in Fig. 1. The $X$-axis is assigned as being parallel to the longest edge of the crystal and $Z$-axis being perpendicular to the basal plane.

The experiments were performed for the fixed value of $X . Z$-value of $0.1 \mathrm{~mm}$ (the distance of the probe to the sample surface) was changed by the adjusting screw (1). The shift of the ESR probe along $Y$-axis was achieved by the micrometer screw (6). The maximum length of scan was equal to $4 \mathrm{~mm}$ and the accuracy of the probe positioning along $Y$-direction was $5 \mu \mathrm{m}$. 
Our experiments were performed using standard X-band EPR spectrometer equipped with an Oxford gas flow cryostat. The measurements were carried out at temperature of $17 \mathrm{~K}$. Too low temperature spoiled the signal making its detection impossible when the probe came closer to the sample.

The procedure of the measurements was following. The crystal in the spectrometer cavity was oriented so that $c$-axis was parallel to the applied DC magnetic field. The probe was adjusted at a given point by fixing appropriate position of both regulated screws. The measurements were run for zero field cooling (ZFC) procedure. The sample was cooled from the temperature higher than the critical one to the temperature of measurement and then magnetic field was switched on relatively fast to the value of $3.30 \mathrm{kOe}$ and then swept throughout the resonance with a rate of $50 \mathrm{Oe} / 16 \mathrm{~s}$. The registration of the ESR signal was performed only once for each cooling procedure.

\section{Results and discussion}

The resonance shift and line width broadening of the EPR-probe line, due to the superconductivity of the sample, are measured as a difference between the resonance field $H_{\mathrm{r}}^{0}$ and line width $\Delta H_{0}$ measured at the temperature of $17 \mathrm{~K}$ in the presence, and without the superconducting sample at a resonance cavity. After lowering the temperature below $T_{\mathrm{c}}$, the ESR probe located above or at the superconductor surface changes its resonance position and line width. The lower is the temperature the larger are these changes. The measured parameters depend on the probe position with respect to the superconducting sample. In Figures 2 and 3 the signal shift and its broadening as a function of the probe position along $Y$ direction for the chosen $X$ and $Z$ values are shown. For illustration the sample location is shown at the bottom of the figures. When the ESR probe is far from the sample, the ESR parameters are approximately close to those expected for a "free" radical. The resonance shift as well as the line width begin to increase sharply, reaching their maximum values in the vicinity of the sample edges. In turn, close to the sample center both parameters slow down exhibiting wide plateau with values near zero. For the whole studied geometry ESR signal was shifted to lower field which indicates that at any studied point over the sample plane and beyond of it the magnetic field was larger than the external applied field $H_{0}$. The variation of the ESR shift from point-to-point reflects the magnetic field distribution around the superconductor.

Theoretical calculations of the field distribution near the flat, long superconducting strip with the magnetic field direction perpendicular to the plane of sample have been reported by Brandt [10]. Generally, the residual magnetic field distribution can be described by the three main contributions:

a) the magnetic flux structure inside the superconductor when $H>H_{\mathrm{cl}}$,

b) magnetic fields induced by the shielding critical currents at the surface of superconducting sample,

c) demagnetizing fields caused by the superconducting sample magnetization $M$. In the superconducting state of the sample the demagnetizing contribution $\left(\Delta H_{\text {loc }}=-4 \pi\left(1-N_{\text {loc }}\right) M\right)$ should always shift the probe resonance field towards 


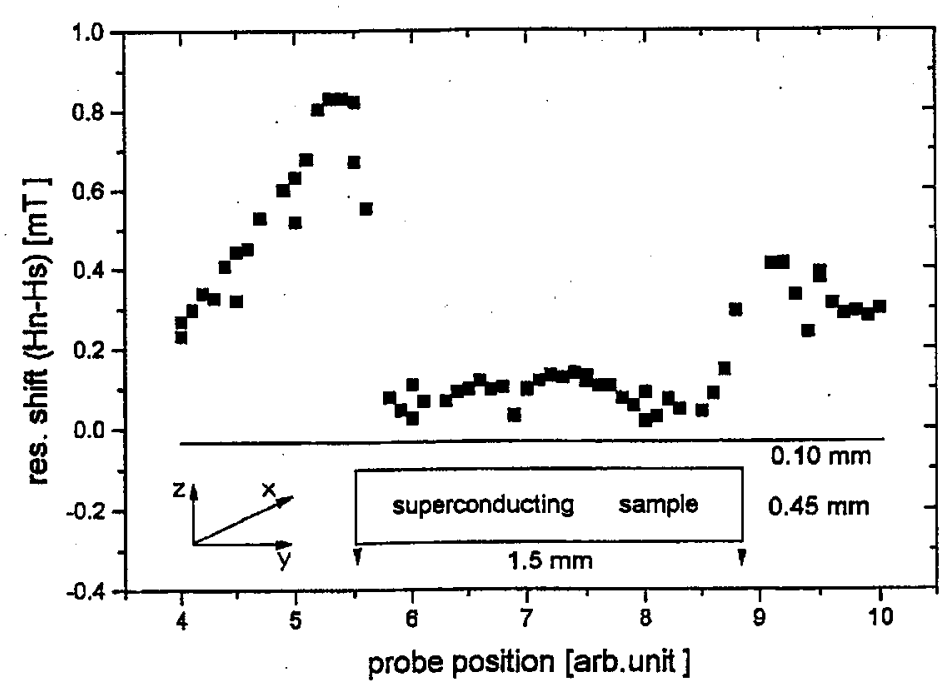

Fig. 2. Dependence of the resonance line shift of the ESR probe on its position over the superconducting Bi2212 strip at $17 \mathrm{~K}$ and $3.3 \mathrm{kOe}$ (position in the micrometer screw units).

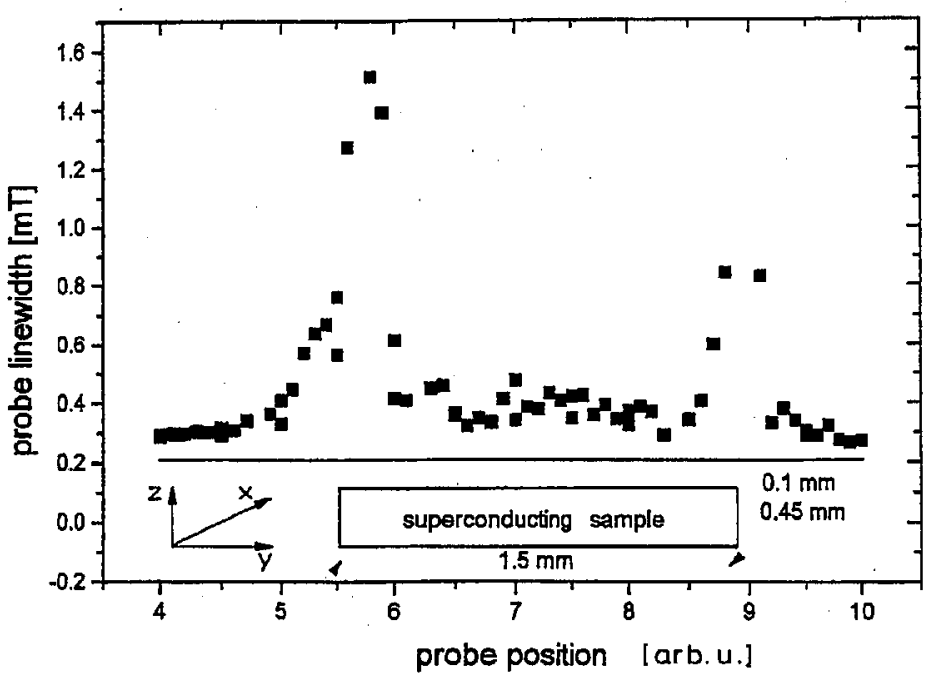

Fig. 3. The line width of the ESR probe as a function of its position around the superconducting Bi2212 strip at $17 \mathrm{~K}$ and magnetic field of $3.3 \mathrm{kOe}$.

higher intensities, since magnetization $M$ is negative. As follows from Fig. 2, for the configuration studied the dentagnetizing field is overcome by other contribution and residual resonance field is shifted towards lower field values. Our results confirm Brandt's prediction that the most dramatic inhomogeneities of the magnetic induction take place just at the edges of the flat superconducting strip when 
external magnetic field is perpendicular. to the main plane of the sample. The largest gradient of the magnetic field in $Y$-direction was estimated to be about $20 \mathrm{kOe} / \mathrm{m}$, and coincides with a point where the line width reaches its maximum value. To study the microscopy contribution in the field distribution we are planning to carry out measurements like that but putting the probe as close as possible to the sample surface since the amplitude of the magnetic field inhomogeneities is smeared exponentially as a function of the distance $Z$ and the characteristic scale $L$ of order of the strip width. Our measurements confirm the great ability of EPR tomography to study subtle profile of magnetic field distribution around superconducting strips or perhaps wires, too.

\section{Acknowledgment}

This work was supported by the Committee for Scientific Research (Poland) under grant No. 2P03B 02110.

\section{References}

[1] G.J. Dolan, G.V. Chandrashekhar, T.R. Dinger, F. Holtzberg, Phys. Rev. Lett. 62, 827 (1989).

[2] M.R. Koblischka, N. Moser, B. Begenheïmer, H. Kronmüller, Physica C 166, 36 (1990).

[3] H.B. Brom, H. Alloul, Physica C 177, 297 (1991).

[4] B. Pümpin, H. Keller, W. Kündig, W. Odermatt, J.M. Savić, J.W. Schneider, H. Simmler, P. Zimmermann, E. Kaldis, S. Rusiecki, Y. Maeno, C. Rossel, Phys. Rev. B 42, 8019 (1990).

[5] Z. Frait, D. Fraitova, E. Pollert, L. Pûst, Phys. Status Solidi B 146, K119 (1988).

[6] B. Rakvin, M. Poźek, A. Dulćić, Solid State Commun. 72, 199 (1990).

[7] N. Bontemps, D. Davidov, P. Monod, R. Even, Phys. Rev. B 43, 11512 (1991).

[8] B. Rakvin, M. Poźek, A. Dulćić, Physica C 170, 166 (1990).

[9] R.I. Khasanov, Yu.I. Talanov, Yu.M. Vashakidze, G.B. Teitel'baum, Physica $C$ 242, 333 (1995).

[10] E.H. Brandt, Phys. Rev. B 46, 8628 (1992). 